\title{
PROCESSO E FORNO PARA PRODUÇÃO DE FERRO GUSA UTILIZANDO MICRO-ONDAS*
}

\section{Resumo}

\author{
Edmilson Renato de Castro ${ }^{1}$ \\ Luiz Alberto Jermolovicius ${ }^{2}$ \\ Marcelo Breda Mourão $0^{3}$ \\ Eduardo Vitor Pouzada \\ Renata Borges do Nascimento 5 \\ Cyro Takano ${ }^{6}$ \\ José Thomaz Senise
}

Apresentam-se o processo e o protótipo de forno para a produção de ferro gusa por redução carbotérmica estimulada por micro-ondas de minério de ferro, na forma de pelotas autoredutoras. O forno de redução com potência regulável de até $15 \mathrm{~kW}$ de micro-ondas de 2.45 $\mathrm{GHz}$ foi especialmente projetado e construído para este processo. A estrutura do forno é em aço inoxidável e utiliza três geradores de micro-ondas focados para o centro de um aplicador cilíndrico. Com esta configuração conseguiu-se produzir um campo eletromagnético de alta densidade energética. A carga de pelotas auto-redutoras, com massa em torno de $20 \mathrm{~g}$ foi reduzida em um cadinho de carbeto de silício. A montagem do forno dispõe de uma balança elétrica adaptada para suportar o cadinho de redução. $O$ presente forno dispõe de controle e medida da potência fornecida e refletida, permitindo medir a energia de micro-ondas efetivamente aplicada à carga. Esta característica permitiu realizar balanços de massa e de energia além de determinar a evolução da taxa de reação através da medida contínua da massa das pelotas sob redução, ao longo do tempo de processamento. Obtiveram-se curvas cinéticas de redução carbotérmica de minério de ferro incentivada por micro-ondas e de seu consumo de energia durante o processo.

Palavras-chave: Ferro gusa; Micro-ondas; Forno redução carbotérmica

\section{PROCESS AND FURNACE FOR PIG IRON PRODUCTION WITH MICROWAVES Abstract}

We present a process and a prototype of furnace for production of pig iron by carbothermic reduction of iron ore in the form of self - reducing pellets enhanced by microwaves. The reduction furnace with adjustable up to $15 \mathrm{~kW}$ of microwave power of $2.45 \mathrm{GHz}$ has been specially designed and built for this purpose. The furnace structure is made of stainless steel and has three microwave generators focused to the center of a cylindrical applicator. With this setup we were able to produce a high energy density electromagnetic field. The burden of self - reducing pellets with mass around $20 \mathrm{~g}$ was reduced in a crucible silicon carbide. The oven features an electric balance appropriately adapted to withstand the crucible reduction. This oven allows the measurement and control of power supplied and reflected establishing the effective energy applied to load. This feature allowed for mass and energy balances and to observe the evolution of reduction rate by continuously measuring of reacting mass during its processing. Curves for carbothermal reduction of iron ore were determined and, also, the energy consumption during the process.

Keywords: Pig iron; Microwave; Carbothermic Reduction furnace.

1 Engenheiro Químico, Mestre, Professor, Laboratório de Micro-ondas, Centro Universitário Instituto Mauá de Tecnologia, São Caetano do Sul, SP, Brasil.

2 Engenheiro Químico, Doutor, Professor, Laboratório de Micro-ondas, Centro Universitário Instituto Mauá de Tecnologia, São Caetano do Sul, SP, Brasil.

3 Engenheiro Metalúrgico, Doutor, Professor, Departamento de Metalurgia e Materiais, Universidade de São Paulo, São Paulo, SP, Brasil.

4 Engenheiro Elétrico, Doutor, Professor, Laboratório de Micro-ondas, Centro Universitário Instituto Mauá de Tecnologia, São Caetano do Sul, SP, Brasil.

5 Engenheira Química, Mestre, Professora, Laboratório de Micro-ondas, Centro Universitário Instituto Mauá de Tecnologia, São Caetano do Sul, SP Brasil.

6 Engenheiro Metalúrgico, Doutor, Professor, Departamento de Metalurgia e Materiais, Universidade de São Paulo, São Paulo, SP, Brasil.

7 Engenheiro Elétrico, Doutor, Professor, Laboratório de Micro-ondas, Centro Universitário Instituto Mauá de Tecnologia, São Caetano do Sul, SP, Brasil.

\footnotetext{
* Contribuição técnica ao 44 Seminário de Redução de Minério de Ferro e Matérias-primas, 15ํ Simpósio Brasileiro de Minério de Ferro e $2^{\circ}$ Simpósio Brasileiro de Aglomeração de Minério de Ferro, 15 a 18 de setembro de 2014, Belo Horizonte, MG, Brasil. 


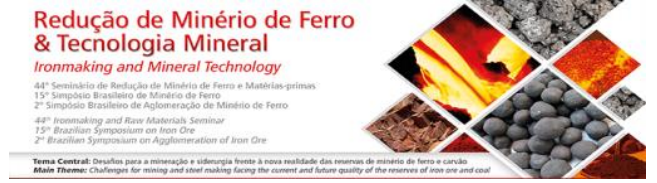

\section{INTRODUÇÃO}

O ferro gusa fundido é usualmente produzido em altos-fornos. Notadamente, a partir de pelotas de minério de ferro, coque ou outra fonte de carbono como redutor e calcário. Entretanto, o alto forno convencional para a produção de ferro gusa líquido necessita de 6 horas ou mais e uma temperatura de aproximadamente $1600^{\circ} \mathrm{C}$, e, infelizmente, não é eficiente energeticamente [1]. Além disso, devido à diminuição de recursos energéticos e a introdução de leis ambientais mais rigorosas, a busca de novos processos de produção de aço e ferro-gusa tornaram-se estratégicos. A redução da emissão de gás carbônico $\left(\mathrm{CO}_{2}\right)$ bem como a conservação de energia são tarefas cruciais para prevenir o aquecimento global [2]. Esta premência ocasiona a busca por um processo de redução que supere estes inconvenientes do alto-forno. Almeja-se, ainda, que a transferência de calor, que desempenha um papel fundamental na velocidade global do processo, seja mais rápida que no forno convencional. Apesar da tecnologia do alto forno ser avançada, novos processos de fabricação do ferro baseados em alternativas inovadoras podem ser introduzidas.

Em estudo anterior, os pesquisadores desenvolveram um equipamento em escala reduzida com finalidade de produzir ferro gusa por redução carbotérmica de minério de ferro por um material carbonoso na forma de pelotas auto redutoras utilizando micro-ondas. Os resultados obtidos nesse primeiro projeto confirmaram a hipótese de que as micro-ondas efetivamente apresentam um aumento da produtividade na redução do minério de ferro [3].

O objetivo deste trabalho é desenvolver um processo e forno em escala ampliada, visando contribuir para a evolução da tecnologia de aplicação de micro-ondas a processos de obtenção de ferro primário com alta eficiência energética, usando micro-ondas como fonte de calor.

\subsection{Conceitos sobre Micro-ondas}

Micro-ondas são radiações eletromagnéticas na faixa de frequência de 0,3 a 300 $\mathrm{GHz}$, com o correspondente intervalo de comprimento de onda de $1 \mathrm{~m}$ a $1 \mathrm{~mm}$. As micro-ondas, tal como as ondas visíveis, obedecem às leis da óptica e podem ser transmitidas, absorvidas ou refletidas dependendo do tipo de material.

Existem basicamente três tipos de materiais, com relação à forma de interação com as micro-ondas. Os materiais transparentes às micro-ondas permitem a total passagem das ondas através de si, sem perdas significativas de energia. Os materiais opacos refletem as ondas eletromagnéticas enquanto que os materiais com altas perdas dielétricas, ou seja, materiais que absorvam irradiação de microondas, também chamados de dielétricos interagem com as micro-ondas absorvendo-as eficientemente, resultando em seu aquecimento [4-5].

A frequência de micro-ondas mais utilizada, dentre as permitidas, pela União Internacional de Telecomunicações (UIT), para aplicação industrial em aquecimento industrial é $2.45 \mathrm{GHz}$. Esta opção é guiada pela melhor relação custo-benefício dos equipamentos geradores de micro-ondas, com relação às demais frequências alocadas para aplicações industriais, científicas e médicas definidas. Esta frequência corresponde ao comprimento de onda no espaço livre de $12,25 \mathrm{~cm}$.

\footnotetext{
* Contribuição técnica ao 44 Seminário de Redução de Minério de Ferro e Matérias-primas, $15^{\circ}$ Simpósio Brasileiro de Minério de Ferro e 2ํ Simpósio Brasileiro de Aglomeração de Minério de Ferro, 15 a 18 de setembro de 2014, Belo Horizonte, MG, Brasil. 


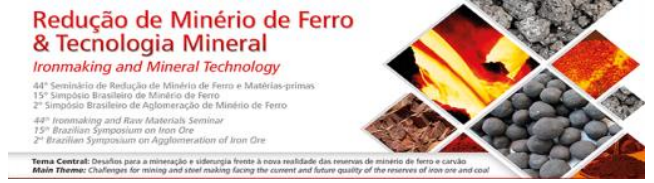

\section{MATERIAIS E MÉTODOS}

\subsection{Construção do Forno de Micro-ondas}

O forno é constituído por uma cavidade cilíndrica em aço inoxidável e utiliza três geradores de micro-ondas focados para o centro deste aplicador. Guias de ondas transferem a energia de micro-ondas destes geradores, dois com potência regulável de até $6 \mathrm{~kW}$ cada e um de $3 \mathrm{~kW}$, para o interior da cavidade podendo totalizar $15 \mathrm{~kW}$. Os trechos dos guias de ondas próximos ao reator cilíndrico estão envolvidos por trocadores de calor para resfria-los durante o processo de redução. Um circulador de micro-ondas é utilizado para proteger cada gerador contra reflexão de energia pelo sistema irradiado. Além destes, há dois acopladores direcionais, por gerador, que permitem medir a potência de micro-ondas irradiada (a que sai do gerador) e a refletida (a que retorna do forno e é direcionada a carga de dissipação). A medida do valor da potência é feita por meio de dois medidores de potência que registram as potências citadas. Com esta configuração consegue-se produzir um campo eletromagnético de alta densidade energética no interior do forno. Filtros que evitam vazamento de micro-ondas estão localizados tanto na parte superior como inferior do forno. O superior assegura a saída do gás inerte (argônio) e dos fumos de reação. O inferior possibilita a passagem de um suporte para o cadinho de carbeto de silício usado para a redução. Este suporte é constituído por um eixo e um prato de porcelana e é acoplado sobre uma balança permitindo monitorar a perda de massa das pelotas em redução no interior de um cadinho. Tanto o prato como o eixo são construídos em material transparente a micro-ondas. A figura 1 mostra uma visão global do equipamento.

A posição do cadinho no interior da cavidade foi otimizada por meio de simulação multifísica empregando o programa COMSOL Multiphysics. A figura 2 apresenta uma imagem da simulação da região com maior densidade energética $\left(\mathrm{W} / \mathrm{m}^{3}\right)$ no interior da cavidade. A cor vermelha indica uma maior temperatura e a azul uma menor.

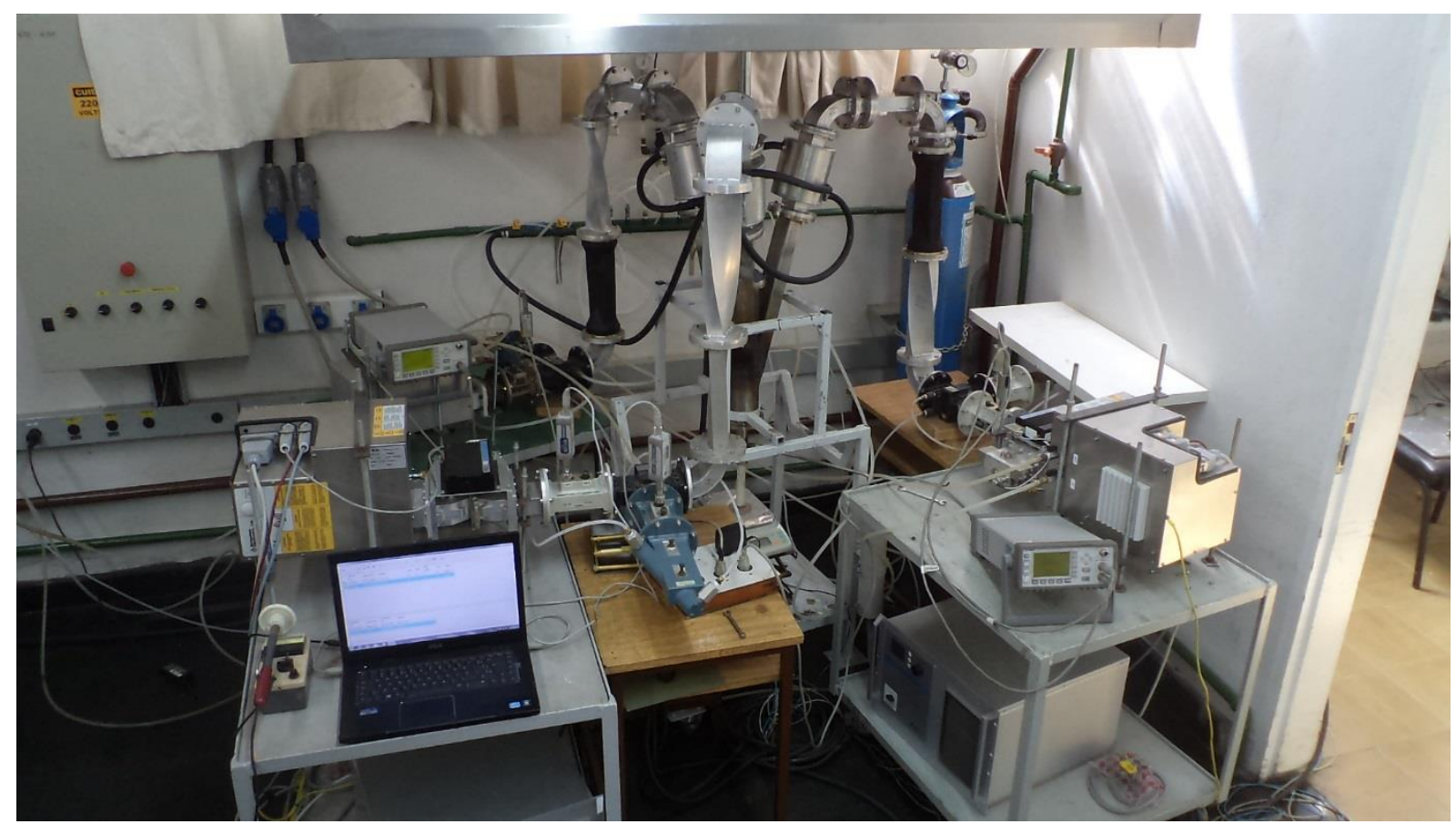

Figura 1. Forno para redução de minério de ferro por irradiação de micro-ondas.

\footnotetext{
* Contribuição técnica ao $44^{\circ}$ Seminário de Redução de Minério de Ferro e Matérias-primas, 15ํ Simpósio Brasileiro de Minério de Ferro e $2^{\circ}$ Simpósio Brasileiro de Aglomeração de Minério de Ferro, 15 a 18 de setembro de 2014, Belo Horizonte, MG, Brasil.
} 
perda de peso. O cálculo da quantidade de material que reagiu durante cada experimento realizado foi efetuado empregando-se a equação 1 :

$$
T R=\left(\frac{M_{i}-M_{t}}{M_{i}-M}\right) \times 100
$$

Onde:

$T R=$ Taxa de reação $(\%)$;

$M_{i}=$ Peso inicial da amostra (g);

$M_{t}=$ Peso no instante $\mathrm{t}(\mathrm{g})$;

$M=$ Porcentagem máxima de perda de peso (teoricamente no valor de $35 \%$ )

\section{RESULTADOS E DISCUSSÃO}

É apresentado os principais resultados encontrados nos ensaios realizados em duplicata para a redução carbotérmica de $20 \mathrm{~g}$ de pelotas auto-redutoras com irradiação por micro-ondas com $4.5 \mathrm{~kW}$ a $2.45 \mathrm{GHz}$.

A figura 3 apresenta a porcentagem de reação em função do tempo de exposição à irradiação por micro-ondas, calculada através da equação 1 .

A figura 4 mostra o perfil da evolução da potência refletida de micro-ondas utilizada na redução carbotérmica de $20 \mathrm{~g}$ de pelotas auto redutoras.

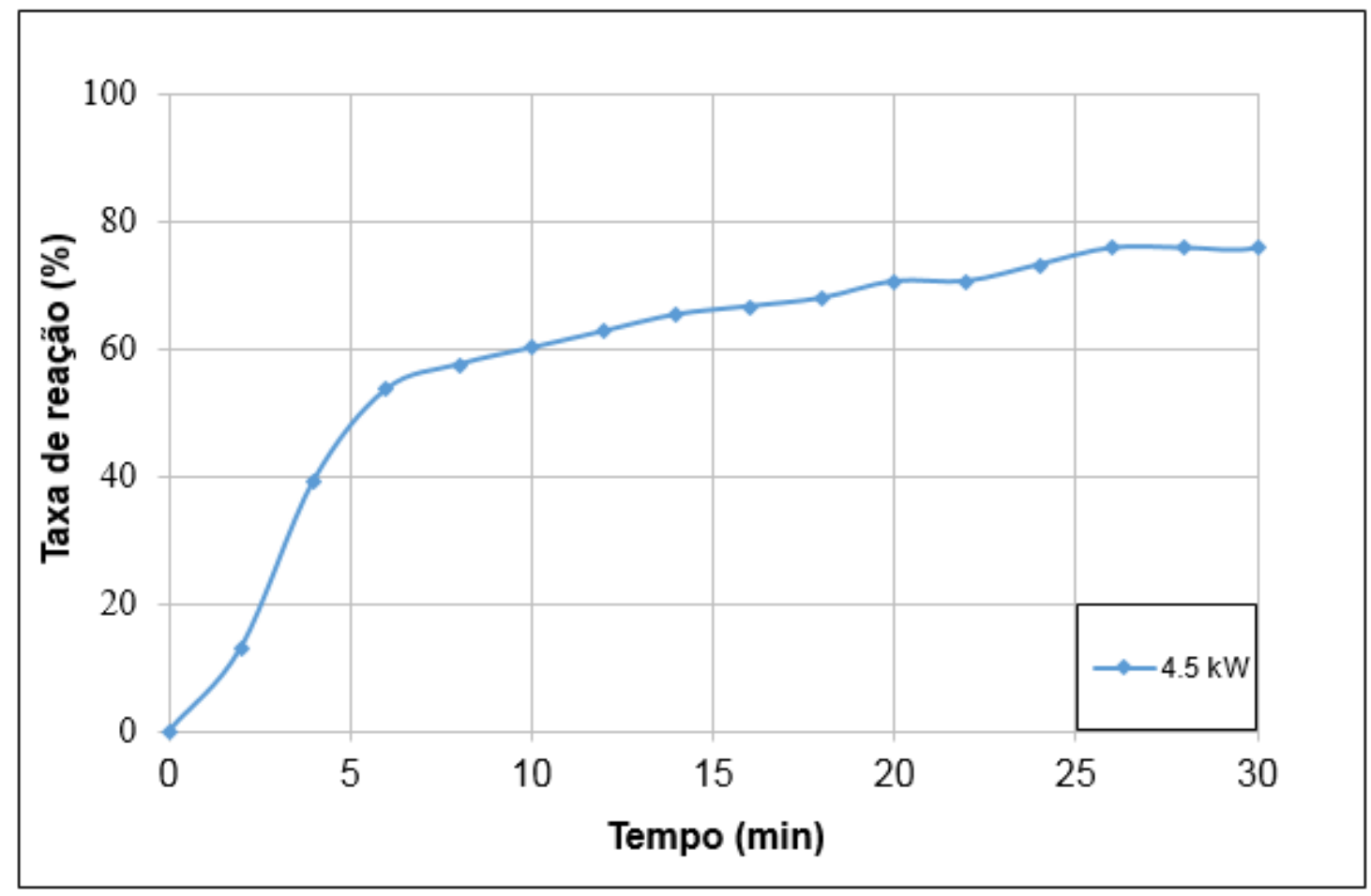

Figura 3. Taxa de reação em função do tempo de irradiação.

* Contribuição técnica ao 44 Seminário de Redução de Minério de Ferro e Matérias-primas, $15^{\circ}$ Simpósio Brasileiro de Minério de Ferro e $2^{\circ}$ Simpósio Brasileiro de Aglomeração de Minério de Ferro, 15 a 18 de setembro de 2014, Belo Horizonte, MG, Brasil. 


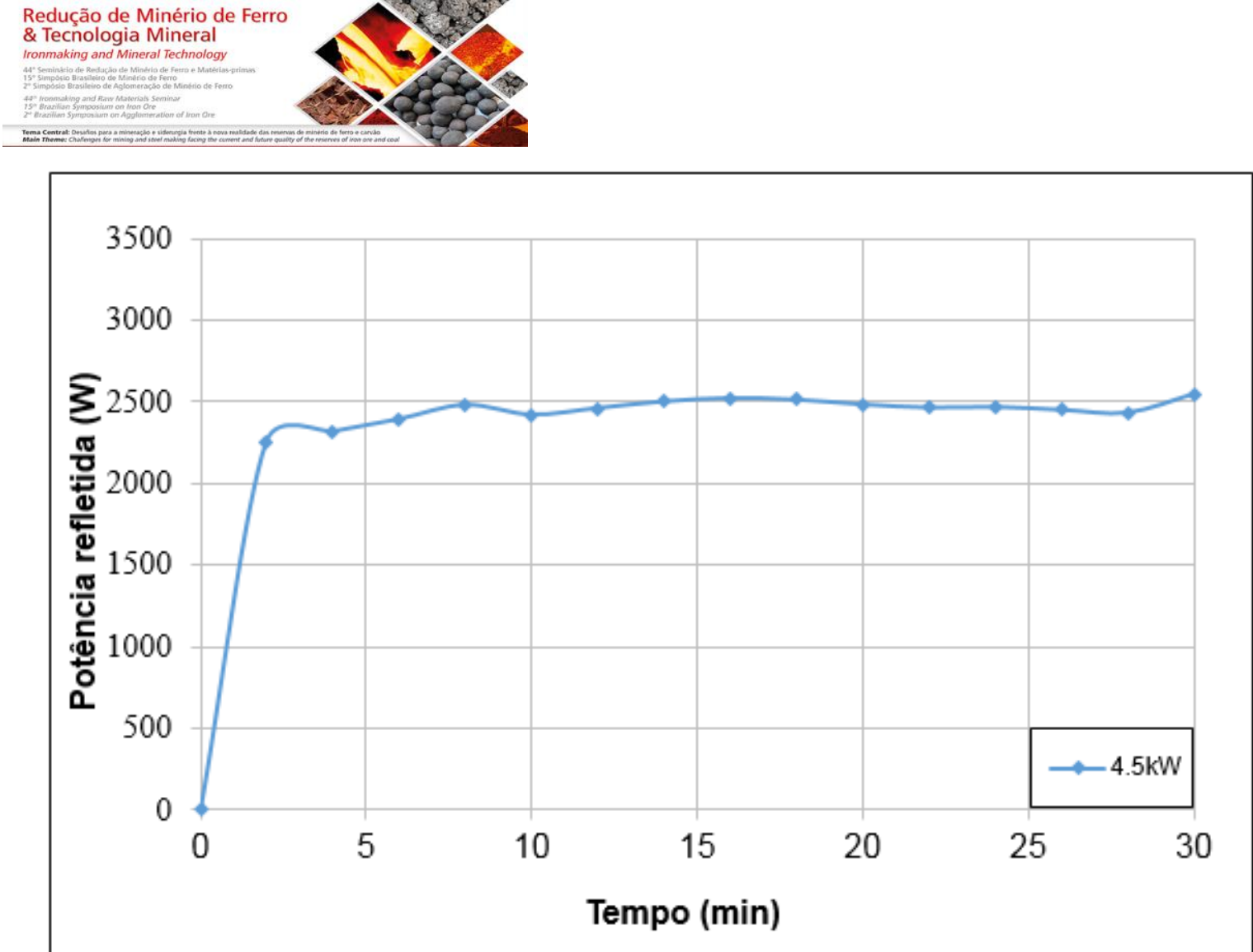

Figura 4. Perfil da evolução da potência de micro-ondas refletida (W) em função do tempo de irradiação.

A taxa de reação apresentada pela figura 3 não atingiu completa redução carbotérmica. Isto se deve provavelmente a alguns fatores, como: a baixa absorção de energia de micro-ondas pelo cadinho. Apesar dele possuir certa quantidade de carbeto de silício que possui elevada constante dielétrica propiciando uma melhor interação das micro-ondas com o material, sua geometria além de sua compactação provocaram uma alta energia refletida como visto através da figura 4 . Isto foi facilmente identificado ao analisar a carga após o ensaio. As pelotas próximas a boca do cadinho estavam totalmente reduzidas. Já as pelotas localizadas no fundo do cadinho continham apenas alguns pontos metálicos em sua superfície.

Um problema encontrado nos ensaios foi a formação de arco voltaico na superfície externa do cadinho. Quando tal fenômeno ocorria o ensaio era interrompido por medida de precaução para não danificar o equipamento. Outro problema encontrado foi a perda térmica pelas paredes do forno devido à falta de isolação térmica.

\section{CONCLUSÃO}

O equipamento ora apresentado abre a oportunidade de uma série de experimentos que permitirão um melhor conhecimento da aplicação das micro-ondas ao sistema cadinho/pelotas de minério de ferro/coque. Este experimento mostrou a necessidade de estudar melhor a interação da energia de micro-ondas com 0 material utilizado na formulação de cadinhos metalúrgicos bem como sua forma geométrica.

\section{Agradecimentos}

Os autores agradecem à colaboração de Dimitri Zinizopoulos Passeti, Murilo Henrique Araujo Elloy, Carlos Tognela, Adriano Gimenez. Agradecem ao Centro Universitário Instituto Mauá de Tecnologia, à Universidade de São Paulo, à FAPESP (projeto 10/51269-1) e à Vale pelo suporte deste trabalho.

* Contribuição técnica ao $44^{\circ}$ Seminário de Redução de Minério de Ferro e Matérias-primas, $15^{\circ}$ Simpósio Brasileiro de Minério de Ferro e 2ํ Simpósio Brasileiro de Aglomeração de Minério de Ferro, 15 a 18 de setembro de 2014, Belo Horizonte, MG, Brasil. 


\section{REFERÊNCIAS}

1 Hara K, Hayashi M, Sato M, Nagata K. Continuous pig iron making by microwave heating with $12.5 \mathrm{~kW}$ at $2.45 \mathrm{GHz}$. Journal of Microwave Power and Electromagnetic Energy, 2011; 45 (3): 137-147.

2 Kashimura K, Nagata K, Motoyasu S. Concept of furnace for metal refining by microwave heating - A design of microwave smelting furnace with low $\mathrm{CO}_{2}$ emission. Materials Transactions, 2010; 51: 1847-1853.

3 Castro ER et al. Forno aprimorado para estudos de processos de redução carbotérmica de minérios incentivada por micro-ondas. Tecnologia em Metalurgia, Materiais e Mineração, 2009; 5(3): 156-161.

4 Sutton WH. Microwave processing of ceramic materials. Ceramic Bulletin, 1989; 68(2): 376-386.

5 Haque KE. Microwave energy for mineral treatment processes - a brief review. International Journal of Mineral Processing, 1999; 57: 1-24.

* Contribuição técnica ao 44ํㅗㄴ Seminário de Redução de Minério de Ferro e Matérias-primas, $15^{\circ}$ Simpósio Brasileiro de Minério de Ferro e 2ํ Simpósio Brasileiro de Aglomeração de Minério de Ferro, 15 a 18 de setembro de 2014, Belo Horizonte, MG, Brasil. 